\title{
Idealized walking and running gaits minimize work
}

\author{
By Manoj SRINIVASAN ${ }^{1,2} \dagger$ AND ANDy Ruina ${ }^{2}$ \\ ${ }^{1}$ Mechanical and Aerospace Engineering, Princeton University, \\ Princeton, NJ 08544, USA \\ ${ }^{2}$ Theoretical and Applied Mechanics, Cornell University, Ithaca, NY 14853, USA
}

Even though human legs allow a wide repertoire of movements, when people travel by foot they mostly use one of two locomotor patterns, namely walking and running. This selection of two from the plethora of options might be because walking and running require less metabolic energy than other more unusual gaits. We addressed this possibility previously using numerical optimization of a minimal mathematical model of a biped (Srinivasan and Ruina, 2006). We had found that for a given steplength the two classical descriptions of walking and running, "inverted pendulum walking" and "impulsive running", do indeed minimize the amount of positive work required at low and high speeds, respectively. Here, for the case of small step lengths, we establish the previous results analytically. First, we simplify the two-dimensional particle trajectory problem to a one-dimensional "elevator" problem. Then we use elementary geometric arguments on the resulting phase plane to show optimality of the two gaits: walking at low speeds and running at high speeds.

Keywords: legged locomotion, energy, optimality, inverted pendulum walking, impulsive running, optimal control

\section{Introduction}

When people travel by foot from point A to point B, and are not rushed, they walk. When they are in a hurry, they run. Classically, human walking has been idealized as a gait in which the hip (or the body center of mass) travels in a series of inverted-pendulum circular arcs about the foot on the ground as shown in figure 1a (e.g., Alexander 1976). In one energy-consistent version of this idealization, only one foot is on the ground at a time, and the transition from one circular arc to the next is accomplished by the trailing leg pushing off just before the heel of the leading leg strikes the ground (Kuo 2001, Ruina et al 2005, Srinivasan and Ruina 2006). Analogously, as shown in figure 1b, running is simply idealized as a series of parabolic free-flights of the body, interrupted by brief bounces. In idealized running the work absorbed in the downward part of a bounce is regenerated on the way back up (Rashevsky 1944, Ruina et al 2005, Srinivasan and Ruina 2006). Among the essentially infinite variety of motions that our two legs are capable of, why do we choose to walk and run in ways that are somewhat close to these two idealizations?

A common and common-sense conjecture is that humans and other animals do things in a way that minimizes metabolic energy expenditure (e.g., Borelli 1680,

$\dagger$ Author for correspondence (msriniva@princeton.edu). 
a) Inverted pendulum walking

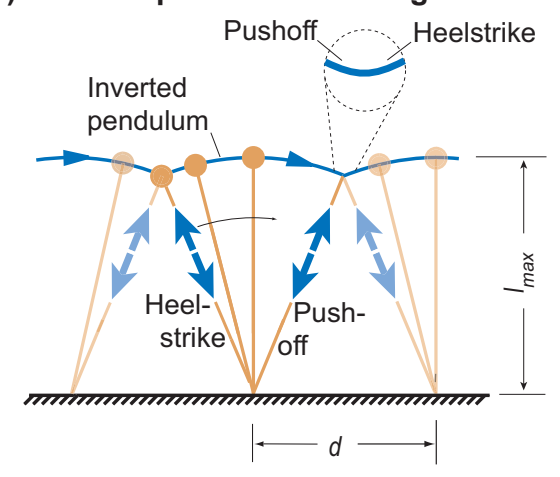

b) Impulsive running

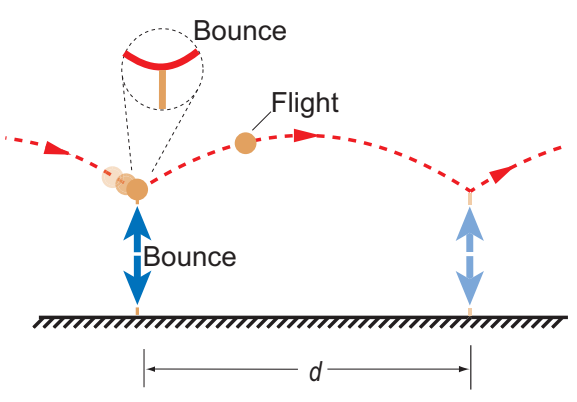

Figure 1. Idealized motions for a) walking and b) running. a) In inverted-pendulum walking, the body vaults in circular arcs on a straight leg. One leg is on the ground at a time. At the change from one circular arc to the next the trailing leg pushes off and then the leading leg heel strikes the ground. b) In impulsive running, the body travels from one parabolic arc to the next with a bounce in between. Adapted from Srinivasan and Ruina (2006) and Srinivasan (2006).

Alexander 1989, Anderson and Pandy 1999). To explore this conjecture, we previously formulated a simple mathematical model of a bipedal animal consisting of a point-mass upper body and two massless legs. The legs can do and absorb work by extending and contracting (Srinivasan and Ruina, 2006, see figure 2). The model was a simplification of earlier models used by Alexander (Alexander, 1980, 1992) and Minetti (Minetti and Alexander, 1997). Using numerical optimization, we found the gaits that minimized the amount of positive work required to travel with a given speed, step length and maximum-allowed leg extension. We found that, indeed, inverted pendulum walking is energetically optimal at low speeds and step-lengths and impulsive running is energetically optimal at higher speeds.

In this paper, we examine the same model, this time analytically, for the special case of small step lengths. Our aims are to more firmly establish the previous numerical result and also to reveal more of the essential structure of the bipedal work-minimization problem.

In section 2, we present problem $\mathrm{A}$, the gait-optimization problem and add some symmetry assumptions. In section 3, we present problem B, the simplification to small step-lengths, further discussed in section 4 . In section 5 , we simplify the locomotion problem to problem $\mathrm{C}$, that of riding a descending elevator given some constraints. Finally, in section 6 , we show that the energy-optimal ways to ride the elevator correspond, in problem A, to inverted pendulum walking and impulsive running.

\section{Problem A: model and symmetry assumptions}

(a) Biped model and metabolic cost

The minimal biped model (figure 2) has a point-mass upper body and two massless legs. Each leg is a telescoping actuator that can change its length. The legs can apply force and perform work on the upper body when they are in contact with 

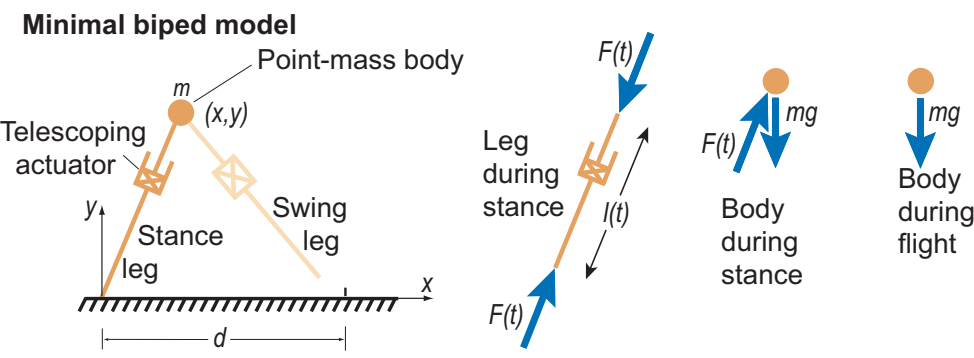

Figure 2. Minimal biped model. The upper body is modeled as a point-mass. The two legs are modeled as massless telescoping actuators, capable of changing length, exerting force and performing work on the upper body. Figure adapted from Srinivasan and Ruina (2006) and Srinivasan (2006).

the ground. When not in contact with the ground, the legs can be swung around arbitrarily. We assume that at most one of the two legs is in contact with ground at a time. In our previous numerical work we enforced a limit on the length of the leg $l_{\max }$. Here, to simplify the mathematical argument, we replace the maximum leg-length constraint with a specification of the height of the center of mass at midstep $l_{0}$. For all problems, the point of contact of the foot with the ground is $x_{c}=0$ throughout the duration of the step under consideration. A step is defined as half a stride. The flight phase, if it exists for a given gait, is the portion of the contact phase in which the force is zero.

The idealized gaits such as inverted pendulum walking and impulsive running, discussed in section 1 , require infinite forces as they involve instantaneous velocity changes corresponding to the kinks in the center of mass trajectories. Because unbounded forces can be troublesome analytically, we impose simple bounds on the leg force $F_{A}(t): F_{\min } \leq F_{A}(t) \leq F_{\max }$, whenever it is convenient to do so. However, allowing infinite forces frees the model of the above force-bound parameters, making the results simpler and more general. We assume that the forces are bounded until the end of section 5 where we allow impulsive forces.

We model the metabolic cost as being separable into three parts.

1. Animals have a positive metabolic expenditure even at rest. Related to this cost, we posit a constant metabolic rate (per unit time) term $\dot{C}_{\text {rest }}$.

2. Leg-swing cost: legged locomotion may require swinging the leg with active muscle work. Here, we assume that this leg-swing metabolic cost $C_{\text {swing }}$ per step is purely a function of the speed and step length or, equivalently, of speed and frequency (Alexander 1976, Kuo 2001).

3. Stance cost: stance cost is incurred by a leg when it is in stance phase, that is, in contact with the ground. We model this cost $\left(C_{\text {stance }}\right.$ per step) as being proportional to a weighted sum of the total positive work and the total negative work performed by the telescoping actuator leg.

If the average forward (horizontal) speed over a step is $v$, the step length is $d_{\text {step }}$, and the step period is $t_{\text {step }}=d_{\text {step }} / v$, the total metabolic $\operatorname{cost} C$ per step is:

$$
C=\dot{C}_{\text {rest }} t_{\text {step }}+C_{\text {swing }}\left(v, d_{\text {step }}\right)+C_{\text {stance }}
$$


In the calculation here we prescribe the forward speed $v$ and the step length $d_{\text {step }}$. Step length is the horizontal distance covered during a step, which may include flight phases $\uparrow$. So it does not matter whether we minimize the energetic cost per step $C$, the energetic cost per unit distance $C / d_{\text {step }}$, or the energetic cost per unit time $C v / d_{\text {step. }}$. All of those minimizations result in the same optimal solution. Further the first two terms in the energy-cost equation 2.1 drop out of the optimization because they are constant for a given forward speed $v$ and step length $d_{\text {step }}$. We observe the role of varying $v$ and $d_{\text {step }}$ by seeing how the optimal solutions depend on them.

The leg's instantaneous mechanical power output is $P=F i$, where $F$ is the leg force, with compression taken as positive, and $l$ is the leg length (figure 2). The stance cost over a step (with duration $-t_{\text {step }} / 2 \leq t \leq t_{\text {step }} / 2$ ) is given by

$$
C_{\text {stance }}=\int_{-t_{\text {step }} / 2}^{t_{\text {step }} / 2}\left(b_{1}[P]^{+}+b_{2}[P]^{-}\right) d t,
$$

where $[P]^{+}$registers positive leg power, equaling $P$ when $P \geq 0$ and equaling zero when $P<0$. $[P]^{-} \equiv[-P]^{+}$registers negative leg power. Typically muscles are assumed to have $b_{1}>0$ (work costs) and $b_{2}>0$ (absorbing mechanical work also has a metabolic cost) but here we only need that $b_{1}+b_{2}>0$.

\section{(b) Symmetry assumptions}

We assume periodic gaits with each step like the next. To simplify the analytic argument here, we assume that the trajectory of the center of mass within a step is symmetric about mid-step (figure 3a). "Mid-step" (at $t=0$ ) is when the body is directly above the foot-contact point. So only half a step - from mid-step till the end of the step - contains all information about the gait. Although this symmetry assumption limits the generality of the analytic demonstration we note that all the numerical optima we previously found had this symmetry about midstep (Srinivasan and Ruina, 2006).

\section{(c) Direct implications of the model assumptions}

The periodicity assumption, for level-ground locomotion, implies that there is no energy change from one midstep to the next. The boundedness of the leg force $F_{A}(t)$ implies that the acceleration is always bounded and that the velocity of the body is continuous. Because the velocity vector along the gait-trajectory is continuous, the symmetry conditions above basically require that the vertical component of the velocity vanishes both at mid-step and at the end of the step (figure $3 \mathrm{~b}$ ).

Because there are no passive dissipation mechanisms, the net work of the legs in one step is zero: $\int P d t=\int\left([P]^{+}-[P]^{-}\right) d t=0$ and the net positive work equals the net negative work, $\int[P]^{+} d t=\int[P]^{-} d t$. Therefore, the cost in equation 2.2 is $C_{\text {stance }}=\left(b_{1}+b_{2}\right) \int[P]^{+} d t$, and proportional to the total positive work. That is, minimizing a weighted sum of the positive and negative work is equivalent to minimizing either the total positive work or the total negative work and does not depend on the values of $b_{1}$ and $b_{2}$ (so long as $b_{1}+b_{2}>0$ ).

$\dagger$ In running "step length" includes the horizontal distance covered while one foot is in contact with the ground in addition to the distance traveled in flight. 


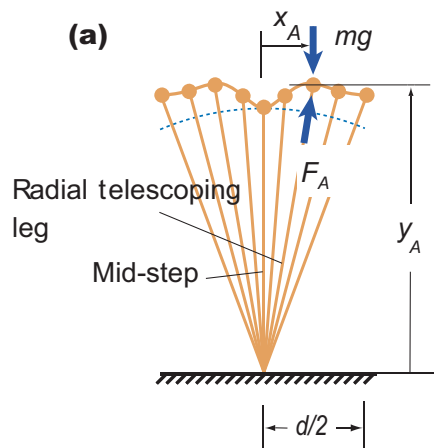

(b)

Figure 3. Problem A (a) One step of a gait that is symmetric about the mid-step. (b) Assuming periodic steps that are symmetric about mid-step implies that the vertical component of the velocity is zero at mid-step and the end of the step.

Secondly, the symmetry assumption implies that the amount of positive work in the first half of the stride equals the amount of negative work in the second half of the stride, and vice versa. So the total cost from equation 2.2 can be rewritten as

$$
\begin{aligned}
C_{A} & =\int_{-t_{\text {step }} / 2}^{0}\left(b_{1}[P]^{+}+b_{2}[P]^{-}\right) d t+\int_{0}^{t_{\text {step }} / 2}\left(b_{1}[P]^{+}+b_{2}[P]^{-}\right) d t \\
& =\int_{0}^{t_{\text {step }} / 2}\left(b_{1}[P]^{-}+b_{2}[P]^{+}\right) d t+\int_{0}^{t_{\text {step }} / 2}\left(b_{1}[P]^{+}+b_{2}[P]^{-}\right) d t \\
& =\left(b_{1}+b_{2}\right) \int_{0}^{t_{\text {step }} / 2}\left([P]^{-}+[P]^{+}\right) d t
\end{aligned}
$$

Without loss of generality, we take $b_{1}+b_{2}=1$, so the cost to be minimized is the sum of the positive and the negative work over half a step: $C_{A}=\int_{0}^{t_{\text {step }} / 2}\left([P]^{-}+[P]^{+}\right) d t$.

\section{(d) Optimization problem $A$}

The position of the center of mass is given by $\left(x_{A}(t), y_{A}(t)\right)$ with mid-step at $x_{A}(0)=0$ (the subscript ' $A$ ' denotes problem $\mathrm{A}$ ).

We wish to determine that values of $\dot{x}_{A}(0)$ and $F_{A}(t)$ over $t \in\left[0, t_{\text {step }} / 2\right]$ that together minimize the total $\operatorname{cost} C_{A}$ :

$$
C_{A}=\int_{0}^{t_{\text {step }} / 2}\left(\left[F_{A} \dot{l}_{A}\right]^{+}+\left[F_{A} \dot{l}_{A}\right]^{-}\right) d t
$$

subject to the restriction that $x_{A}, y_{A}, l_{A}=\sqrt{x_{A}^{2}+y_{A}^{2}}$, and $F_{A}$ all obey the differential equations

$$
\begin{aligned}
& m \ddot{x}_{A}=F_{A} \frac{x_{A}}{l_{A}}, \text { and } \\
& m \ddot{y}_{A}=-m g+F_{A} \frac{y_{A}}{l_{A}}
\end{aligned}
$$

and the boundary conditions that $x_{A}(0)=0, y_{A}(0)=l_{0}, x_{A}\left(t_{\text {step }} / 2\right)=d_{\text {step }} / 2$, $d y_{A} /\left.d t\right|_{0}=0$ and $d y_{A} /\left.d t\right|_{t_{\text {step }} / 2}=0$. 
The specification of the initial conditions and the single control function legforce $F_{A}(t)$ determine $\left(x_{A}(t), y_{A}(t)\right)$ through the differential equation. We proceed to solve this optimization problem, with the added "small angle" assumption that $d \ll l_{0}$ by considering a sequence of equivalent replacement problems.

Lemma 2.1. Trajectories can be completely specified by $d_{\text {step }}, t_{\text {step }}$, and $\ddot{y}_{A}(t)$

Proof. Since $y_{A}(0)=l_{0}, \dot{y}_{A}(0)=0$, we can obtain the vertical velocity component $\dot{y}_{A}(t)$ and the vertical position $y_{A}(t)$ by integrating the vertical acceleration $\ddot{y}_{A}$. Having determined $y_{A}(t)$, we can determine $F_{A}(t) / l_{A}(t)$ from equation 2.6:

$$
\frac{F_{A}(t)}{l_{A}(t)}=\frac{m \ddot{y}_{A}(t)+m g}{y_{A}(t)} \stackrel{\text { def }}{=} f(t)
$$

We can use this $f(t)$ in equation 2.5 to obtain for $x_{A}$ :

$$
m \ddot{x}_{A}=f(t) x_{A} .
$$

Solving for $x_{A}(t)$ requires two initial conditions. One of these, the horizontal position at mid-step, is given: $x_{A}(0)=0$. The other initial condition $\dot{x}_{A}(0)$ can uniquely be determined as a function of $f(t), d_{\text {step }}$ and $t_{\text {step }}$ as follows.

First, observe that equation 2.8 is linear in $x_{A}$. This linearity implies that the final horizontal position is given by $x_{A}\left(\frac{t_{\text {step }}}{2}\right)=k_{1} x_{A}(0)+k_{2} \dot{x}_{A}(0)$, where $k_{1,2}$ depend on $f(t)$. Since $x_{A}(0)=0$, we have $x_{A}\left(\frac{t_{\text {step }}}{2}\right)=k_{2} \dot{x}_{A}(0)$. This proportionality implies that any given $d_{\text {step }}=2 x_{A}\left(\frac{t_{\text {step }}}{2}\right)$ is obtained by a unique choice of the initial speed $\dot{x}_{A}(0)$. Determination of $x_{A}(t)$ completes the description of the trajectory.

\section{Problem B: Riding on a circular track with a vertical extendible leg}

Now we simplify problem A to obtain problem B, motivated by the following observations.

1. In problem A, motion in circular arcs about the foot contact point do not require mechanical work $\left(P=F_{A} \dot{l}_{A}=0\right.$ since $\left.i_{A}=0\right)$. We would like a similar property for problem B.

2. We have assumed that the leg makes small angles with the vertical.

We combine these two ideas to obtain problem B (figure 4a). Imagine a circular track of radius $l_{0}$ centered at $(0,0)$ in the sagittal plane and a point-mass body riding the circular track with a vertical telescoping leg. The foot of the vertical telescoping leg rides on the circular track with constant horizontal speed. This constant horizontal velocity component is assumed to be enforced externally, without any energetic cost to the biped.

As shown in figure $4 \mathrm{a}, y_{B}$ is the vertical position of the point-mass from the ground. $y_{V}(t)$ is the length of the vertical telescoping leg and $y_{C}(t)=y_{B}(t)-y_{V}(t)$ is the height of the circular track from the ground, corresponding to where the pointmass is at time $t . F_{B}$ is the vertical force transmitted by the vertical telescoping actuator. 


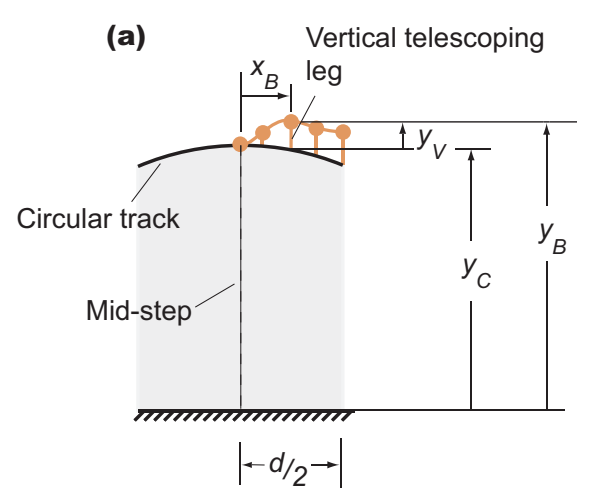

(b)

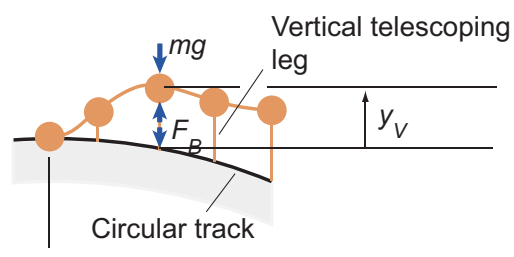

Figure 4. Problem B: riding a circular track (a) The radial telescoping leg of problem A has been replaced by a vertical telescoping leg in Problem B. The foot of the vertical telescoping leg moves on the circular track with constant horizontal speed, enforced externally. (b) The action of the vertical telescoping leg riding on a circular track is shown in detail. The vertical velocity components must vanish at mid-step and end of step.

\section{(a) Optimization problem $B$}

The governing equation for the vertical motion is:

$$
m \ddot{y}_{B}=m\left(\ddot{y}_{V}+\ddot{y}_{C}\right)=F_{B}-m g .
$$

where the vertical force $F_{B}(t)$ is bounded: $F_{\min } \leq F_{B}(t) \leq F_{\max }$. The goal is to minimize the sum of the positive and negative work $C_{B}$ of the vertical telescoping actuator:

$$
C_{B}=\int_{0}^{t_{\text {step }} / 2}\left[F_{B}(t) \dot{y}_{B}(t)\right]^{+}+\left[F_{B}(t) \dot{y}_{B}(t)\right]^{-} d t
$$

subject to the same boundary conditions as problem A:

$$
y_{B}(0)=l_{0}, d y_{B} / d t(0)=0 \text {, and } d y_{B} / d t\left(t_{\text {step }} / 2\right)=0 .
$$

The one extra constraint in problem A, namely $x_{A}\left(t_{\text {step }} / 2\right)=d_{\text {step }} / 2$ is automatically satisfied here by the assumption of constant horizontal speed $v$. A formula for $\ddot{y}_{C}$ in equation 3.1 might be obtained by twice differentiating $y_{C}=\sqrt{l_{0}^{2}-x_{B}^{2}}$ with respect to time, where $x_{B}(t)=v t$.

\section{Small step lengths: Problem B is similar to Problem A}

An optimization problem is characterized by the objective function to be minimized and the constraints defining the space of feasible solutions. Here, we will only show the similarity of the objective functions in problems A and B rigorously, and provide only a heuristic justification for the similarity of the set of feasible trajectories.

\section{(a) Definition: analogous trajectories}

As established in lemma 2.1, every trajectory in Problem A is completely described by the specification of the speed $v$, step length $d_{\text {step }}$ and the vertical acceleration $\ddot{y}_{A}(t)$. For every such trajectory in Problem A, we define a unique analogous 
trajectory in Problem B as that having the same speed $v$, step length $d_{\text {step }}$ and vertical motion $y_{B}(t)=y_{A}(t)$. A trajectory in problem $\mathrm{B}$ can be uniquely described thus.

(b) Relation between the space of feasible trajectories in Problems $A$ and $B$

The vertical forces are equal for analogous trajectories in problem $\mathrm{A}$ and $\mathrm{B}$ because the vertical accelerations are the same, by definition. Therefore, $\left|F_{B}(t)\right|=$ $\left|F_{A y}\right| \leq\left|F_{A}(t)\right|$. As a consequence of this inequality and the boundedness of the forces, the feasible region of problem $\mathrm{A}$ is not in general identical to the feasible region of Problem B. However, we imagine that the disparity between the two feasible sets vanishes in some sense in the limit of small step lengths and as we let the force bounds go to their respective infinities. We shall not state this claim more precisely nor prove it rigorously.

\section{(c) A one parameter family of gaits: limit of small step-lengths}

In order to meaningfully take the limit of small step lengths, we need to specify what is kept constant as we take the limit. In Problem A, given a particular trajectory with speed $v$, step-length $d_{0}$, step-duration $t_{0}$ and vertical acceleration $\ddot{y}_{A 0}(t)=g(t)$, we can generate a one-parameter family of gaits, all with the same average speed $v$, but with different step-lengths by simply using a time-stretched version of the vertical acceleration $g(t)$. That is, the vertical acceleration for a gait with a step-length $d_{\text {step }} \neq d_{0}$ is given by

$$
\ddot{y}_{A}(t)=g\left(t d_{0} / d_{s t e p}\right)
$$

Clearly, this one parameter family is uniquely defined for every $v, d_{0}$, and $g(t)$. Having defined a one parameter family of gaits parameterized by the step length, we can meaningfully ask how the energetic costs scale as $d_{\text {step }} \rightarrow 0$. In the following, we use $d_{\text {step }}=\epsilon$, as $\epsilon \rightarrow 0$.

Proposition 4.1. For analogous trajectories of Problems $A$ and $B$, we have $C_{A} / C_{B}=$ $1+O\left(\epsilon^{2}\right)$ when $d_{\text {step }}=\epsilon$. That is, we claim that

$$
\int_{0}^{t_{\text {step }} / 2}\left[F_{A}(t) \dot{l}_{A}\right]^{+} d t=\left(1+O\left(\epsilon^{2}\right)\right) \int_{0}^{t_{\text {step }} / 2}\left[F_{B}(t) \dot{y}_{V}\right]^{+} d t
$$

for analogous trajectories of Problems $A$ and $B$.

Simply showing that $C_{A} \rightarrow C_{B}$ is not meaningful as both $C_{A}$ and $C_{B}$ approach zero as $d \rightarrow 0$.

Proof. Noting that the integrands in equation 4.2 are essentially products of two terms, we shall simply show that the analogous multiplicands in the integrands are respectively equal up to a factor of at most $\left(1+O\left(\epsilon^{2}\right)\right)$. In particular, lemma 4.4 shows that $\dot{l}_{A}=\left(1+O\left(\epsilon^{2}\right)\right) \dot{y}_{V}$ and lemma 4.5 shows that $F_{A}=\left(1+O\left(\epsilon^{2}\right)\right) F_{B}$ for all analogous trajectories. We now show lemmas 4.2 and 4.3 , which will be used in lemma 4.4. 
Lemma 4.2. In problem $A$, for any given $\ddot{y}_{A 0}(t)=g(t)$ and $v$, the fluctuations in the leg-length are much smaller than the initial leg-length in the limit of small step-lengths. That is, $l_{A}(t)=l_{0}\left(1+O\left(\epsilon^{2}\right)\right)$.

Proof. Noting that $\ddot{y}_{A}(t)=g\left(t d_{0} / d_{\text {step }}\right)$ from Eq.4.1, we have

$$
y_{A}(t)=l_{0}+\int_{0}^{t_{\text {step }}} \int_{0}^{t^{\prime}} g\left(\frac{t^{\prime \prime} d_{0}}{d_{\text {step }}}\right) d t^{\prime \prime} d t^{\prime}=l_{0}+O\left(\epsilon^{2}\right),
$$

because $t^{\prime} \leq t_{\text {step }}=O(\epsilon)$ and $g(t)$ is bounded and independent of $d_{\text {step }}$. Combining this with $x_{A}(t) \leq d_{\text {step }}$ and $l_{A}(t)=\sqrt{y_{A}^{2}+x_{A}^{2}}$, we have $l_{A}(t)=l_{0}+O\left(\epsilon^{2}\right)=$ $l_{0}\left(1+O\left(\epsilon^{2}\right)\right)$.

Lemma 4.3. In problem $A$, for any given $g(t)$ and $v$, the fluctuations of the horizontal velocity component become much smaller than the average horizontal speed in the limit of small step-lengths. In particular, $\dot{x}_{A}(t)=v\left(1+O\left(\epsilon^{2}\right)\right)$.

Proof. $g(t)$ determines $y_{A}(t)$, which in turn determines $f(t)$ in the differential equation for $\dot{x}_{A}(t)$ : $\ddot{x}_{A}=f(t) x_{A}$ (equation 2.8). This gives $\dot{x}_{A}(t)=\dot{x}_{A}(0)+$ $\int_{0}^{t} f\left(t^{\prime}\right) x_{A}\left(t^{\prime}\right) d t^{\prime}=\dot{x}_{A}(0)+O\left(\epsilon^{2}\right)$ since both $t$ and $x_{A}(t)$ are $O(\epsilon)$. That is, the fluctuations in the horizontal speed about the initial speed $\dot{x}_{A}(0)$ are $O\left(\epsilon^{2}\right)$. But this implies $\dot{x}_{A}(t)=v+O\left(\epsilon^{2}\right)$. Since $v$ does not depend on $\epsilon$, we have $\dot{x}_{A}(t)=$ $v\left(1+O\left(\epsilon^{2}\right)\right)$.

Lemma 4.4. In problems $A$ and $B, \dot{l}_{A}=\dot{y}_{V}\left(1+O\left(\epsilon^{2}\right)\right)$ for analogous trajectories. That is, the leg's extension rate in problem $A$ is asymptotically equal to the extension rate of the vertical telescoping leg in problem $B$.

Proof. 1. $l_{A}=\sqrt{x_{A}^{2}+y_{A}^{2}}$. Differentiating this equation with respect to $t$, we have $\dot{l}_{A}=x_{A} \dot{x}_{A} / l_{A}+y_{A} \dot{y}_{A} / l_{A}$. Noting that $l_{A}=l_{0}\left(1+O\left(\epsilon^{2}\right)\right)=y_{A}\left(1+O\left(\epsilon^{2}\right)\right)$ from lemma 4.2 , we have $\dot{l}_{A}=\left(x_{A} \dot{x}_{A} / l_{0}+\dot{y}_{A}\right)\left(1+O\left(\epsilon^{2}\right)\right)$. Equivalently, $\dot{y}_{A}=\left(\dot{l}_{A}-x_{A} \dot{x}_{A} / l_{0}\right)\left(1+O\left(\epsilon^{2}\right)\right)$

2. The equation for the circular track of radius $l_{0}$ is $y_{C}^{2}=l_{0}{ }^{2}-x_{B}^{2}$. This implies $\dot{y}_{C}=-\dot{x}_{B} x_{B} / y_{C}$. Since $y_{C}=l_{0}\left(1+O\left(\epsilon^{2}\right)\right)$, we have $\dot{y}_{C}=-(1+$ $\left.O\left(\epsilon^{2}\right)\right) x_{B} \dot{x}_{B} / l_{0}$.

3. $y_{V}=y_{B}-y_{C}$ and $y_{B}=y_{A}$ for analogous trajectories. Therefore, $\dot{y}_{V}=$ $\dot{y}_{B}-\dot{y}_{C}=\dot{y}_{A}-\dot{y}_{C}$. Substituting the results of items (1) and (2) above into this equation, we have

$$
\dot{y}_{V}=\left(1+O\left(\epsilon^{2}\right)\right)\left(\dot{l}_{A}-\frac{x_{A} \dot{x}_{A}}{l_{0}}+\frac{x_{B} \dot{x}_{B}}{l_{0}}\right)
$$

But by lemma 4.3 , we have $\dot{x}_{A}(t)=v\left(1+O\left(\epsilon^{2}\right)\right)=\dot{x}_{B}\left(1+O\left(\epsilon^{2}\right)\right)$ and as a corollary, $x_{A}(t)=x_{B}(t)\left(1+O\left(\epsilon^{2}\right)\right)$. Using these, we have

$$
\dot{y}_{V}=\left(1+O\left(\epsilon^{2}\right)\right)\left(\dot{l}_{A}+\frac{x_{B} \dot{x}_{B}\left(1+O\left(\epsilon^{2}\right)\right)^{2}}{l_{0}}-\frac{x_{B} \dot{x}_{B}}{l_{0}}\right)=\left(1+O\left(\epsilon^{2}\right)\right) \dot{l}_{A}
$$


Lemma 4.5. $F_{A}=F_{B}\left(1+O\left(\epsilon^{2}\right)\right)$

Proof. If $F_{A y}$ is the component of $F_{A}$ in the vertical direction, $F_{A y}=\frac{y_{A} F_{A}}{l_{A}}=$ $\frac{y_{A} F_{A}}{\sqrt{y_{A}^{2}+x_{A}^{2}}}=\left(1+O\left(\epsilon^{2}\right)\right) F_{A}$. By definition, the vertical accelerations and therefore, the vertical forces for analogous trajectories in problems A and B are identical. So $F_{B}=F_{A y}=F_{A}\left(1+O\left(\epsilon^{2}\right)\right)$.

\section{Problem C: Riding an elevator that accelerates down}

(a) Replace the circular path by a parabolic path

In the discussion of the equivalence of problems A and B, only item 2 of lemma 4.4 above uses anything about the foot of the vertical telescoping actuator traveling in a circular path. And $y_{C}(t)$ enters the proof of lemma 4.4 only as its first derivative $\dot{y}_{C}$. Differentiating $y_{C}(t)=\sqrt{l_{0}^{2}-x_{B}^{2}(t)}=\sqrt{l_{0}^{2}-(v t)^{2}}$, we have $\dot{y}_{C}=-v^{2} t / y_{C}$.

We replace the circular track by a parabolic track, $y_{p}(t)=l_{0}-\frac{v^{2} t^{2}}{2 l_{0}}$. We see that $y_{p}(t)==l_{0}-\frac{v^{2} t^{2}}{2 l_{0}}$ describes a parabolic track in the $x$ - $y$ plane by substituting $t=x_{B} / v$ in the expression for $y_{p}(t)$. If the foot of the vertical telescoping actuator travels with constant horizontal speed on this parabolic track, the corresponding vertical velocity component of the foot will be

$$
\dot{y}_{p}=-\frac{v^{2} t}{l_{0}}=-\frac{v^{2} t}{\sqrt{y_{C}^{2}+x_{B}^{2}}}=-\frac{v^{2} t}{\left(1+O\left(\epsilon^{2}\right)\right) y_{C}}=\left(1+O\left(\epsilon^{2}\right)\right) \dot{y}_{C}
$$

So replacing the circle $y_{C}$ by the parabola $y_{p}$ does not falsify lemma 4.4 or proposition 4.1. We make this replacement because the vertical acceleration of the foot $\left(\ddot{y}_{p}=-\frac{v^{2}}{l_{0}}\right)$ is conveniently a constant when traveling on the parabolic track with constant horizontal speed.

Summarizing, the original problem A has been reduced to finding the path of the body that entails the least cost while riding a parabolic track with a vertical telescoping leg and constant horizontal velocity. The constraints are that the initial and final vertical speeds must be zero.

\section{(b) Galilean relativity}

Since the horizontal speed is a constant in problem B, the body will have no horizontal motion when observed from an inertial frame moving with horizontal speed $v$. Since the foot of the vertical telescoping actuator is riding the parabolic arc, when the constant speed is subtracted out, the foot will be simply seen to be accelerating downwards with $-\ddot{y}_{p}=a_{e}$, say.

$$
a_{e}=-\ddot{y}_{p}=v^{2} / l_{0}
$$

Note further that the cost to be minimized (equation 3.2) and the boundary conditions (equation 3.3) also depend on only the vertical motion of the telescoping leg. Therefore, we can rewrite problem B with the parabolic track, just in terms of the vertical coordinates. 


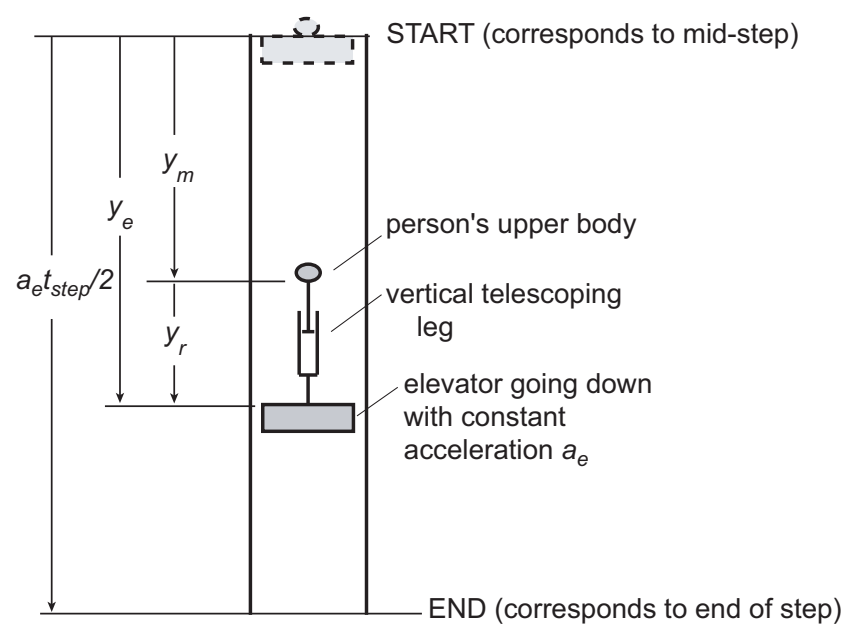

Figure 5. Problem C: Riding an accelerating elevator. Both the point-mass and the elevator start at the same position (START) with zero vertical speeds. The elevator maintains a constant downward acceleration $a_{e}$. The point-mass can push or pull against the elevator using arbitrarily strong vertical telescoping legs. When the elevator reaches END, the vertical speed of the point-mass should again be zero. The objective is to ensure this by doing the least amount of work with the vertical telescoping legs.

\section{(c) Problem C: Elevator problem}

The foot moving downwards with constant downward acceleration $a_{e}=\frac{v^{2}}{l_{0}}$ is most conveniently represented as being attached to an elevator moving downwards with constant acceleration $a_{e}$ (figure 5 ). At time $t=0$, both the person and the elevator are at the same position (without loss of generality). This initial state corresponds to mid-step, the apex of the parabolic or circular arc. The person can push or pull on the elevator with his vertical telescoping legs without affecting the elevator's motion. The person must have zero vertical velocity at time $t=\frac{t_{\text {step }}}{2}$. The objective is to meet this zero vertical velocity constraint by reacting against the elevator in a manner that incurs the least cost.

Figure 5 shows the key variables in the elevator problem. Downward displacements and velocities are considered positive. $y_{e}$ is the position of the elevator, $y_{m}$ is the position of the mass $m$, and $y_{r}=y_{e}-y_{m}$ is the relative position of the elevator with respect to the mass. The vertical motion of the point-mass is governed by the equation: $m \ddot{y}_{m}=m g-F$, where $F$ is the compressive leg-force. The differential equation for the relative position $y_{r}$ is

$$
m \ddot{y}_{r}=m \ddot{y}_{e}-m \ddot{y}_{m}=m a_{e}-m g+F
$$

At mid-step $t=0$, the positions and the velocities of both the elevator and the mass are equal to zero: $y_{e}(0)=y_{m}(0)=y_{r}(0)=0$ and $\dot{y}_{e}(0)=\dot{y}_{m}(0)=\dot{y}_{r}(0)=0$. Thus this starting configuration is represented by the origin $\mathrm{O}$ in the $y_{r}-\dot{y}_{r}$ plane (figure $6 \mathrm{a}, \mathrm{b}, \mathrm{c})$. Since $\ddot{y}_{e}(t)=a_{e}$, we have $\dot{y}_{e}\left(\frac{t_{s t e p}}{2}\right)=\frac{a_{e} t_{\text {step }}}{2}$. The constraint that the mass must have zero velocity at the end of the step $\left(\dot{y}_{m}\left(\frac{t_{\text {step }}}{2}\right)=0\right)$ can be restated as $\dot{y}_{r}\left(\frac{t_{s t e p}}{2}\right)=\dot{y}_{e}-\dot{y}_{m}=\frac{a_{e} t_{s t e p}}{2}$. That is, in the $y_{r}-\dot{y}_{r}$ plane, the final state should lie somewhere on the line $\mathrm{AB}$ corresponding to $\dot{y}_{r}=\frac{a_{e} t_{\text {step }}}{2}$ (figure $6 \mathrm{a}, \mathrm{b}, \mathrm{c}$ ). 
Thus, graphically, the goal in the elevator problem is to take the state of the system from the origin $\mathrm{O}$ in the $y_{r}-\dot{y}_{r}$ plane to any point on the line $\mathrm{AB}$ in a given amount of time $\frac{t_{\text {step }}}{2}$, and with minimum cost.

Summarizing, the elevator problem is to minimize the total cost

$$
C_{e}=\int_{0}^{t_{\text {step }} / 2}\left[F(t) \dot{y}_{r}\right]^{+}+\left[F(t) \dot{y}_{r}\right]^{-} d t
$$

subject to the terminal constraint $\dot{y}_{r}\left(t_{\text {step }} / 2\right)=0$, no explicit constraint on the force $F(t)$, and the relative position $y_{r}(t)$ being determined by the differential equation $m \ddot{y}_{r}=m a_{e}-m g+F$ and the initial conditions $y_{r}(0)=0$ and $\dot{y}_{r}(0)=0$. Recall that $[P]^{+}=P$ when $P \geq 0,[P]^{+}=0$ when $P<0$, and $[P]^{-}=[-P]^{+}$.

Equation 5.4 tells us that work is performed only when there is change in the relative distance between the elevator and the person.

\section{(d) Limit of infinite force-bounds}

As in Srinivasan and Ruina (2006) and as alluded to in section 2a, we formally wish to determine the limit of the sequence of optimal solutions as the force-bounds in the elevator problem increase without bound $\left(F_{\max } \rightarrow \infty\right.$ and $\left.F_{\min } \rightarrow-\infty\right)$ for every combination of speed $v$ and step-length $d_{\text {step }}$. To show the similarity of Problem A with Problem B (section 4) in the limit of small step-lengths, we assumed that the leg-forces were bounded. However, in the following discussion, we find it convenient to allow infinite leg-forces, in particular, impulses that change the vertical speed instantaneously.

\section{Optimal "gaits" in the elevator problem.}

(a) Walking and running in the elevator problem

We now describe how the two idealized gaits, inverted pendulum walking and impulsive running, described earlier in section 1 and illustrated in figure 1, can be most naturally described in the context of the elevator problem. Inverted pendulum walking can be most naturally described as riding the elevator till $t=t_{\text {step }} / 2$ and then pushing-off impulsively against the elevator at exactly $t=t_{\text {step }} / 2$ so that the vertical velocity of the person gets reset to zero. Impulsive running, on the other hand, is jumping impulsively off the elevator at $t=0$ giving the point-mass an initial vertical velocity that ensures that the vertical speed at $t=t_{\text {step }} / 2$ equals zero. Before we show that these strategies are optimal (proposition 6.1), we present some results, to be used later, about the structure of the optimization problem in the $y_{r}-\dot{y}_{r}$ plane.

\section{(b) Constant energy contours}

Rearranging equation 5.3 , we get $F=m\left(\ddot{y}_{r}+g-a_{e}\right)$. The instantaneous mechanical power of this force (as used in equation 5.4 ) is given by:

$$
P=F \dot{y}_{r}=m\left(\ddot{y}_{r}+g-a_{e}\right) \dot{y}_{r}=\frac{d}{d t} \underbrace{\left(m \dot{y}_{r}^{2} / 2+m\left(g-a_{e}\right) y_{r}\right)}_{E}=\frac{d E}{d t}
$$


a) Optimal trajectory: $a_{e}<g$

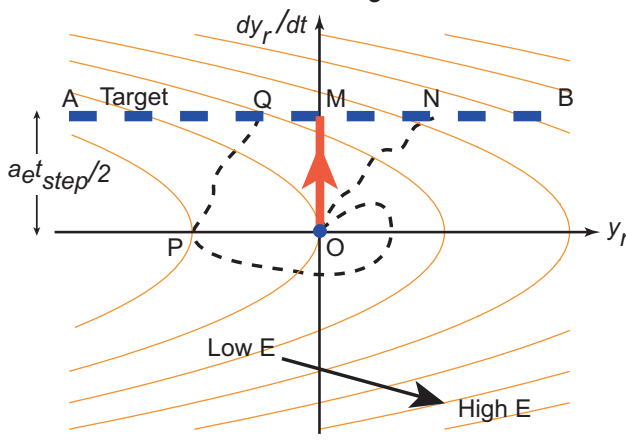

c) Optimal trajectory: $a_{e}=g$

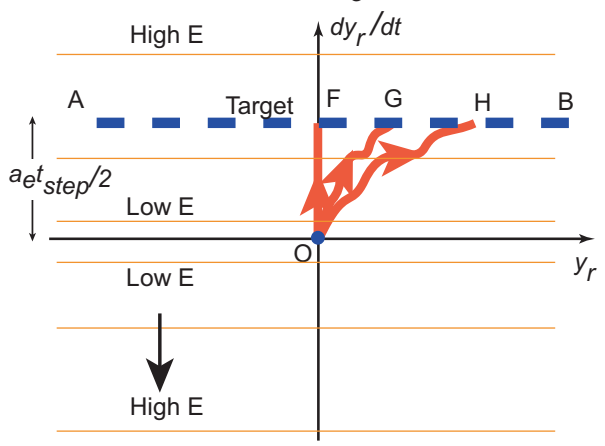

b) Optimal trajectory: $a_{e}>g$

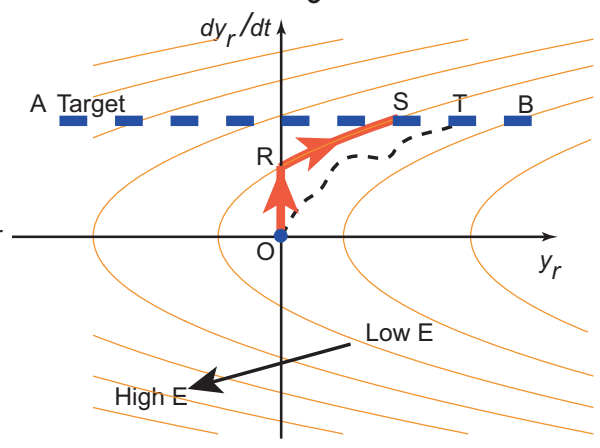

d) Accessible directions

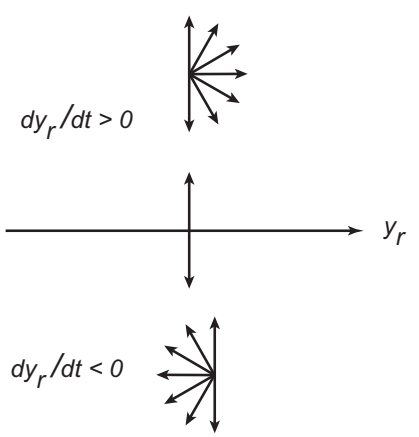

Figure 6. Solution to the elevator problem. (a) Case $1, a_{e}<g$ : The thin parabolic contours are constant energy lines. The goal is to go from the origin $\mathrm{O}$ to the dotted line $\mathrm{AB}$ with the least positive work. Optimal strategy is the vertical line OM. Two alternate suboptimal strategies $\mathrm{ON}$ and $\mathrm{OPQ}$ are shown as thin dashed lines. (b) Case $2, g<a_{e}$ : Optimal strategy is the path ORS. An alternate path OT is shown as a thin dotted line. (c) Case 3, $g=a_{e}$ : All trajectories (e.g., OF, OG, OH) that do not backtrack have the same cost and hence are optimal. (d) shows the set of accessible velocity directions directions in which a trajectory can proceed. In the upper half-plane, the trajectories can never move left. In the lower half plane, the trajectories can never move to the right. No magnitude information is intended by the equality of the length of the arrows.

Equation 6.1 simply notes that the leg power $P$ is the time derivative of the total energy $E=m \dot{y}_{r}^{2} / 2+m\left(g-a_{e}\right) y_{r}$. Contours of constant energy $E$ in the $y_{r^{-}}$ $\dot{y}_{r}$ plane play a key role in the proof of optimality of walking and running below. Generically, the constant energy contours are parabolas in the $y_{r}-\dot{y}_{r}$ plane (figure 6 ). When $a_{e}<g$, the parabolas open leftward (figure 6a), when $a_{e}>g$ the parabolas open rightward (figure $6 \mathrm{~b}$ ), and when $a_{e}=g$, the parabolas degenerate into straight lines (figure $6 \mathrm{c}$ ). The constant energy contours coincide with the trajectories corresponding to gravitational free-fall, with $F(t)=0$ and $P(t)=0$. The total cost $C_{e}$ is simply the absolute sum of all positive and negative increments of $E$ along a trajectory. A trajectory that goes from contour $E_{1}$ to contour $E_{2}$ incurs a cost of at least $\left|E_{1}-E_{2}\right|$. 
(c) Feasible directions of progress in the $y_{r}-\dot{y}_{r}$ plane

The trajectory of the point-mass in the $y_{r}-\dot{y}_{r}$ plane is determined by the following equations (the second equation among which is the same as Equation 5.3).

$$
\frac{d y_{r}}{d t}=\dot{y}_{r} \text { and } \frac{d \dot{y}_{r}}{d t}=a_{e}-g+\frac{F}{m}
$$

The set of feasible tangent vector directions is shown in Fig. 6 d. Since $\dot{y}_{r}>0$ in the upper half-plane, any trajectory can only move to the right. Similarly, a trajectory can move only to the left in the lower half-plane $\left(\dot{y}_{r}>0\right)$. Because the force can be unbounded, all rightward tangent vector directions are accessible in the upper half-plane and all leftward directions are accessible in the lower half-plane. On the line $\dot{y}_{r}=0$, a trajectory necessarily has a vertical tangent.

No information about the feasible magnitudes of the tangent vectors to a trajectory is intended by the equality of arrow lengths in figure $6 \mathrm{~d}$. Not every tangentvector magnitude is possible in a given tangent vector direction (equation 6.2).

(d) Time duration of a given trajectory in the $y_{r}-\dot{y}_{r}$ plane

The time taken to go between two points $\mathrm{P}_{1}$ and $\mathrm{P}_{2}$ along a trajectory in the $y_{r}-\dot{y}_{r}$ plane is given by the integral $\int d t=\int_{P_{1} P_{2}} \frac{d y_{r}}{\dot{y}_{r}}$ if $\dot{y}_{r} \neq 0$ everywhere along the trajectory.

By choosing $F=m g-m a_{e}$ and $\dot{y}_{r}=0$, the right hand sides of the equation 6.2 become identically zero. Thus, for this choice of force, the line $\dot{y}_{r}=0$ becomes a line of fixed points. That is, arbitrary lengths of time can be spent at any point on the $\dot{y}_{r}=0$ line. So if a trajectory $\mathrm{P}_{1} \mathrm{P}_{2}$ intersects the $\dot{y}_{r}=0$ axis, the total time duration for the trajectory needs to be formally calculated as:

$$
\text { Time duration }=\left(\text { Time spent at line } \dot{y}_{r}=0\right)+\left(\int_{P_{1} P_{2}: \dot{y}_{r} \neq 0} \frac{d y_{r}}{\dot{y}_{r}}\right) \text {. }
$$

The specific instances of the improper integral in equation 6.3 that we will consider will either be convergent by construction or their convergence will not affect the ensuing arguments.

Proposition 6.1. For the elevator problem, when $a_{e}<g$, "inverted pendulum walking" minimizes the cost $C_{e}$, when $a_{e}>g$, "impulsive running" minimizes the cost $C_{e}$, and when $a_{e}=g$, there are infinitely many strategies that have the same minimum $\operatorname{cost} C_{e}$.

Case 1: $a_{e}<g$. Recall that the goal is to move from the origin $\mathrm{O}$ to the line $\mathrm{AB}$ with the least cost in figure 6a. We claim that the optimal strategy is to "walk": that is, ride the elevator with zero relative speed $\dot{y}_{r}$ (remaining at the origin $\mathrm{O}$ ) and then at the last moment $t=t_{\text {step }} / 2^{-}$push-off impulsively to make the vertical velocity of the point-mass $\dot{y}_{m}$ zero (jumping instantaneously from $\mathrm{O}$ to $\mathrm{M}$ ).

Proof. By construction, it is clear that OM is a feasible trajectory. We now need to establish that every other feasible trajectory (such as the dotted lines ON and OPQ in figure 6a) necessarily incurs a greater cost. We shall consider two types of feasible trajectories. 
1. Trajectories entirely in the upper half-plane Trajectories entirely in the upper half-plane can never move leftward, as discussed earlier. Starting from $\mathrm{O}$, such a trajectory can reach any point on $\mathrm{AB}$ in the right half-plane $\left(y_{r}=0\right.$ axis included). The point on the right-half of $\mathrm{AB}$ that is on the leastenergy parabolic contour is $\mathrm{M}$. So, for instance, reaching $\mathrm{N}$ requires higher net positive work. The path OM reaches $\mathrm{M}$ by performance of only positive work and so achieves the minimum possible cost $\left|E_{M}-E_{O}\right|$. Further, the only way to reach $\mathrm{M}$ from $\mathrm{O}$ while remaining in the upper half plane (therefore, never going left) is the straight line OM, thus establishing uniqueness of the minimum.

2. Trajectories not entirely in the upper half-plane Consider any such path OPQ, where PQ is the part of the path that lies entirely in the upper half-plane, and $\mathrm{Q}$ is on $\mathrm{AB}$. Then,

$$
\text { Cost } C_{O P Q}=C_{O P}+C_{P Q} \geq C_{O P}+\text { Minimum cost from } \mathrm{P} \text { to } \mathrm{AB}
$$

The cost for going from $\mathrm{P}$ to $\mathrm{AB}$ when restricted to the upper half-plane is minimized when $\mathrm{Q}$ is directly above $\mathrm{P}$ (repeating arguments from item 1 ). This minimum cost will be the same as that of OM, $C_{O M}=\left|E_{M}-E_{O}\right|$ (because the energy-contour parabolas are equally spaced). Thus, $C_{O P Q} \geq C_{O P}+C_{O M}$. If $\mathrm{OP}$ does not degenerate to the point $\mathrm{O}$, the trajectory $\mathrm{OP}$ requires either some positive work or negative work or both, so that the cost associated with OP is positive. Therefore, the cost $C_{O P Q}$ is greater than the cost $C_{O M}$, establishing optimality for OM among all possible trajectories.

Case 2: $a_{e}>g$. We claim that the optimal strategy in this case is to "run". That is, jump impulsively at $t=0^{+}$(going from $\mathrm{O}$ to $\mathrm{R}$ in figure $6 \mathrm{~b}$ ), and then fly freely without doing any further work (constant- $E$ parabola RS with $F=0$ ). Here, R has $\dot{y}_{r}\left(0^{+}\right)=g t_{\text {step }} / 2=-\dot{y}_{m}\left(0^{+}\right)$, so that at $t=t_{\text {step }} / 2, \dot{y}_{m}=0$ and $\dot{y}_{r}=a_{e} t_{\text {step }} / 2$ on $\mathrm{AB}$, as required. So the path RS takes exactly $t=t_{\text {step }} / 2$.

Proof. By construction, the trajectory ORS is feasible (satisfies all constraints). A necessary condition for an alternate path OT to have a lower positive work than ORS is that OT never touches RS or an E-contour of higher energy. So path OT lies entirely below the path ORS (figure $6 \mathrm{~b}$ ). We will now show that any such path OT that has lower positive work will necessarily take longer than $t_{\text {step }} / 2$ to go from $\mathrm{O}$ to $\mathrm{AB}$.

We use the equation 6.3 for the time of a trajectory. By construction of trajectory ORS, the time spent at $\mathrm{O}$ or along $\mathrm{OR}$ is equal to zero. So the time duration for the path ORS is simply given by $\int_{R S} \frac{d y_{r}}{\dot{y}_{r}}$. Since $y_{r}(S)<y_{r}(T)$ and at a given $y_{r}$, $\dot{y}_{r}\left(y_{r}\right)$ on OT (when defined) is less than that on ORS, we have

$$
\int_{0^{+}}^{y_{r}(S)} \frac{d y_{r}}{\dot{y}_{r}}<\int_{0^{+}}^{y_{r}(T)} \frac{d y_{r}}{\dot{y}_{r}} .
$$

Therefore, Time for ORS $<$ Time for $\mathrm{O}^{+} \mathrm{T} \leq$ Time for OT. Summarizing, no path $\mathrm{OT}$ that requires a lower positive work than ORS can also reach $\mathrm{AB}$ in time. 
Therefore, any trajectory that reaches $\mathrm{AB}$ at the same time as ORS must require at least as much positive work.

Cost $C_{O R S}=C_{O R}=\left|E_{R}-E_{O}\right|$ is all due to positive work. Since any other feasible trajectory (satisfying all constraints) requires at least as much positive work, any other feasible trajectory incurs at least as much cost. Thus ORS minimizes the cost. Uniqueness of the minimum ORS is easily demonstrated: any path not identical to ORS but only touching ORS from below and requiring same positive work or cost, will require a longer time, repeating earlier arguments.

Case 3: $a_{e}=g$. For this case, we show below by construction that there are infinitely many globally optimal trajectories.

Proof. Consider trajectories that go from $\mathrm{O}$ to $\mathrm{AB}$ over which $\dot{y}_{r}$ never decreases (e.g., OF, OG, OH in figure 6c). That is, $\ddot{y}_{r} \geq 0$. Such "non-backtracking" trajectories also necessarily have $\dot{y}_{r} \geq 0$. Named examples include inverted pendulum walking, impulsive running, pendular running (Srinivasan and Ruina, 2006), level walking, and many other gaits.

When $a_{e}=g$, the equation of motion reduces to $m \ddot{y}_{r}=F$. For non-backtracking trajectories, first, we need $\ddot{y}_{r} \geq 0$ and therefore $F \geq 0$, that is, non-tensional leg forces. Next, for such a trajectory to reach $\mathrm{AB}$ in $t=t_{\text {step }} / 2$, we need $\int_{0}^{t_{\text {step }} / 2} \frac{F(t)}{m} d t=$ $\frac{e t_{\text {step }}}{2}$. Clearly, infinitely many functions $F(t) \geq 0$ satisfy this equation. Further, when $a_{e}=g$, the power $P$ from equation 6.1 simplifies to $P=m \ddot{y}_{r} \dot{y}_{r}$, which for non-backtracking trajectories is greater than zero by definition. That is, all these trajectories reach $\mathrm{AB}$ by performance of no negative work and only positive work. Since line AB is an iso-energy contour, we see that the cost incurred by every nonbacktracking trajectory equals the energy difference between $\mathrm{O}$ and $\mathrm{AB}$, clearly the minimum possible cost. Summarizing, all feasible gaits with non-tensional leg-forces $(F \geq 0)$ are optimal when $a_{e}=g$.

\section{Discussion}

Recall that the elevator acceleration $a_{e}$ is the centripetal acceleration while traveling on a circular arc: $a_{e}=\frac{v^{2}}{l_{0}}$ from equation 5.2. Thus the conditions for walking $\left(a_{e}<g\right)$, running $\left(a_{e}>g\right)$ and the transition $a_{e}=g$ reduce to conditions on the so-called Froude number $v^{2} /\left(g l_{0}\right)$ (Alexander 1976) or the non-dimensional forward speed $V=v / \sqrt{g l_{0}}$. We have shown that the gait transition (at least for low step lengths) occurs at $V=1$.

One classical theory of why animals switch from an walk to a run (Alexander 1976, Usherwood 2006, Srinivasan 2006) is that traveling in a circular arc requires physically unreasonable tensional forces at higher speeds. This classical theory does indeed show why pendular walking is undesirable at high speeds, but it does not explain why another walking gait, one without such a tightly curved trajectory (like level walking), might not be optimal at higher or lower speeds.

Here, despite allowing for tensional leg forces, we find that all the optimal gaits have only compressive leg forces, and, due purely to energetic reasons, that there is still a gait transition. Interestingly, the speed above which walking requires tension $(V=1)$ and when walking becomes energetically unfavorable coincide, at least for 
small step lengths (see discussion of longer step lengths in Srinivasan and Ruina, 2006).

At the transition speed $V=1$ or $a_{e}=g$, the elevator problem shows that all gaits that use only non-tensional leg forces $(F \geq 0)$ have the same cost. The cost landscape is perfectly flat at the optimum. The flatness of the cost-landscape in this simple model suggests that the actual optimal solution may be very sensitive to small changes in the model, at least near $V=1$. Indeed, numerical optimizations indicate that at slightly larger step-lengths an apparently unique third gait, "a pendular run" seems optimal at these intermediate speeds (Srinivasan and Ruina, 2006). In the calculation here, the pendular run is one of an infinite number of optimal gaits at exactly $V=1$.

The analytic demonstration in this paper depends on the limit of small steplengths. However, the essential structure of the optimal solutions seem to be preserved as we go from small step-lengths to large step-lengths (as seen in the numerical explorations of Srinivasan and Ruina, 2006), except for details near $V=1$.

The allowance of infinite (impulsive) forces was another simplification. Presumably, more realistic finite force bounds will "soften" the impulses, resulting in a smooth change in velocities instead of discontinuous changes. Perhaps the essential structure of the optima will remain the same.

Finally, the biped model itself is obviously a gross simplification of a real bipedal animal, not taking into account, for instance, the presence of elastic elements (tendons that can store and release mechanical energy reducing requirements for muscle work), leg dynamics (except for simple leg swing costs in section 2), muscle properties (including a more realistic metabolic cost not exactly aligned with mechanical work), and other anatomical realism (Anderson and Pandy 1999). Obviously, it is of interest which generalizations of the simple model here change the conclusions, and in what way.

\section{Conclusions}

Legs of real animals perform positive and negative work during locomotion at finite speed. One might hope for ever-improving coordination strategies that could reduce this mechanical work to arbitrarily small levels. The present calculation shows that animal legs cannot get away with zero positive work, even the absence of dissipation, when traveling at finite speeds and step lengths (see also Chatterjee and Garcia 2000 for a related discussion in the context of passive-dynamic robots). We started with a minimal model of a bipedal animal and asked what the energy-optimal ways are for it to travel on its legs. We reduced this locomotion problem to a simpler and somewhat entertaining problem of finding the work-minimizing way of riding a downward accelerating elevator while ensuring that the vertical velocity at the end of the ride is zero. We solved this simpler problem analytically, with elementary geometric arguments. We showed that the optimal solutions for the elevator problem are analogous to the solutions obtained for the original locomotion optimization problem of Srinivasan and Ruina (2006). That is, we discovered that the two classic idealized gaits, inverted pendulum walking and impulsive running, indeed minimize a work-based metabolic cost. That both of these two classic idealizations that have been around for a few decades in various forms should prove to be work-minimizing for the same biped model at different regimes is interesting and is perhaps a tribute 
to those earlier researchers, mainly Rashevsky in the 1940s and Alexander, Cavagna et al, and Margaria in the 1970s (Rashevsky 1948, Alexander 1976, Margaria 1976, Cavagna et al 1977).

This research was supported by an NSF robotics grant to AR (NSF CISE 0413139). MS was supported by NSF FIBR 0425878 during revisions of this manuscript. An anonymous reviewer pointed out a key error in an earlier version of this paper.

\section{References}

Alexander, R. McN. Mechanics of bipedal locomotion., volume 1, pages 493504. Pergamon Press, New York, 1976.

Alexander, R. McN. 1980. Optimum walking techniques for quadrupeds and bipeds. J. Zool., Lond. 192, 97-117.

Alexander, R. McN. 1992. A model of bipedal locomotion on compliant legs. Phil. Trans. R. Soc. Lond. B338, 189-198.

Minetti, A. E. \& Alexander, R. McN. 1997. A Theory of Metabolic Costs for Bipedal Gaits. J. Theor. Biol. 186, 467-476.

Alexander, R. McN. 1989. Optimization and gaits in the locomotion of vertebrates. Physiol. Rev. 69, 1199-1227.

Anderson, F. C. \& Pandy, M. 1999. Dynamic optimization of human walking. J. Biomech. Engg. 123, 381-390.

Borelli, J. A. 1680. On the movement of animals (De Motu Animalium, Pars prima) P. Maquet (trans.) Springer-Verlag, Berlin, p. 152.

Cavagna, G. A., Heglund, N. C., \& Taylor, C. R. 1977. Mechanical work in terrestrial locomotion: two basic mechanisms for minimizing energy expenditure. Am. J. Physiol., $233,243261$.

Chatterjee, A. \& Garcia, M. 2000. Small slope implies low speed in passive dynamic walking. Dynamics and Stability of Systems, 15, 139-157.

Kuo, A. D. 2001. A simple model predicts the step length-speed relationship in human walking. Journal of Biomechanical Engineering, 123: 264-269.

Margaria, R. 1976. Biomechanics and energetics of muscular exercise. Clarendon Press, Oxford.

Srinivasan, M. \& Ruina, A. 2006. Computer optimization of a minimal biped model discovers walking and running. Nature. 439, 72-75.

Srinivasan, M. 2006. Why walk and run: Energetic costs and energetic optimality in simple mechanics-based models of a bipedal animal. Ph.D. thesis, Cornell University.

Rashevsky, N. 1948. On the locomotion of Mammals. Bull. Math. Biophysics. 10, 11-23. 